\title{
Massless Dirac Fermions in a Square Optical Lattice
}

\author{
Jing-Min $\mathrm{Hou}^{1}$ * Wen-Xing Yang ${ }^{1,3}$, and Xiong-Jun Liu ${ }^{2}$ \\ ${ }^{1}$ Department of Physics, Southeast University, Nanjing, 211189, China \\ 2 Department of Physics, Texas A $6 M$ University, College Station, Texas 77843-4242, USA \\ 3 Institute of Photonics Technologies, National Tsing-Hua University, Hsinchu 300, Taiwan
}

(Dated: January 5, 2009)

\begin{abstract}
We propose a novel scheme to simulate and observe massless Dirac fermions with cold atoms in a square optical lattice. A $U(1)$ adiabatic phase is created by two laser beams for the tunneling of atoms between neighbor lattice sites. Properly adjusting the tunneling phase, we find that the energy spectrum has conical points in per Brillouin zone where band crossing occurs. Near these crossing points the quasiparticles and quasiholes can be considered as massless Dirac fermions. Furthermore, the anisotropic effects of massless Dirac fermions are obtained in the present square lattice model. The Dirac fermions as well as the anisotropic behaviors realizeded in our system can be experimentally detected with the Bragg spectroscopy technique.
\end{abstract}

PACS numbers: 37.10.Jk, 03.75.Ss, 05.30.Fk

\section{INTRODUCTION}

Realization of two-dimensional (2D) systems of massless Dirac fermions is of great fundamental importance, in the light of many exotic phenomena obtained in such systems, such as zero modes, fractional statistics, unconventional Landau levels, parity anomaly, chirality, and anomalous quantum Hall effects [1, 2, 3]. However, two-dimensional massless Dirac field have not been observed untill the creation of graphene, a monolayer of graphite [4, 5]. Electrons in graphene, obeying a linear dispersion relation, behave like massless Dirac fermions $[4,5,6,6,8,19,10,11,12]$.

Besides graphene, physicists also make efforts to search for other physical systems, e.g. patterned $2 \mathrm{D}$ electron gases [13] and ultracold atoms in the honeycomb optical lattice [14, 15, 16, 17] , to simulate massless Dirac fermions. Realization of honeycomb optical lattice opens new possibility of studying Dirac fermions in cold atoms which provide an extremely clean environment and controllable fashion unique access to the study of complex physics [18, 19, 20]. Nevertheless, all of the above systems require the hexagonal symmetry. Then, it is very attractive to find a system without the hexagonal symmetry to observe massless Dirac fermions.

Ultracold atom systems provide an ideal platform to study many interesting physics in condensed matters. To investigate the effects of gauge fields with ultracold atoms, several schemes have been proposed to create an artificial Abelian gauge field 21, 22, 23, 24, 25, 26. or a non-Abelian gauge field [27, 28, 29] for neutral atoms with laser fields. Many effects have been studied for cold atoms in an effective gauge field, e.g., Stern-Gerlach effect for chiral molecules 30], Double and negative reflection [31], Landau levels [32], spin Hall effect [33, 34], induced spin-orbit coupling [35], magnetic

*Electronic address: jmhou@seu.edu.cn monopole [36], spin field effect transistors [37]. Furthermore, some groups have realized the light-induced gauge fields in experiments [38, 39].

In this paper, we propose a scheme to generate a staggered gauge field with laser fields. A 2D square lattice model under this artificial gauge field has a spectrum behaving like massless Dirac fermions. Furthermore, our lattice model does not have the hexagonal symmetry. In our scheme, the energy bands of the system exhibit degeneracy points where the conduction and valence bands intersect. Near the these crossing points the dispersion relation is linearly dependent on the momentum, say, is of the Dirac type. The present scheme suggests a new direction to study Dirac fermions in the optical lattice without the hexagonal symmetry. (a)

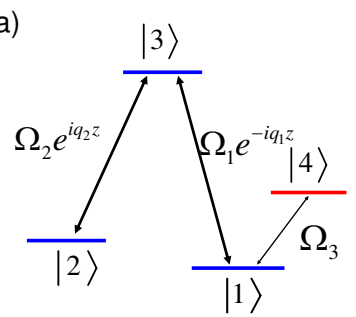

(c)

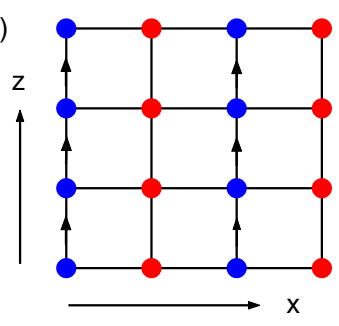

(b)

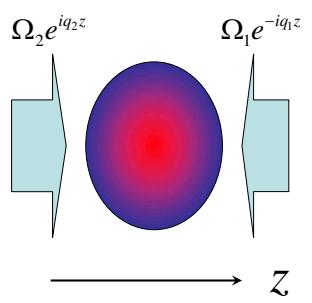

(d)

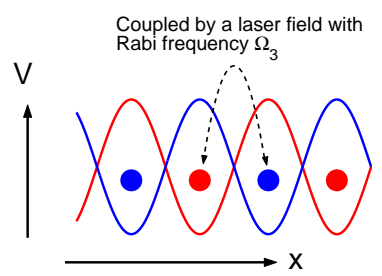

FIG. 1: (a) The atomic levels and the interactions between atoms and laser fields. (b) Schematic representation of the experimental setup with the two laser beams incident on the cloud of atoms. (c) Schematic of the square optical lattice and the designed phase factor (denoted by arrows). (d) The scheme of overlapping the two state-selective optical lattices. 


\section{MODEL}

We consider a system of ultracold fermionic atoms with four levels shown in FIG 1. (a). This atomic level configuration can be experimentally realized with alkali atom ${ }^{6} \mathrm{Li}$ [40]. We choose the atomic states $2 S_{1 / 2}\left(F=1 / 2, m_{F}=\right.$ $1 / 2), 2 S_{1 / 2}\left(F=3 / 2, m_{F}=3 / 2\right), 2 P_{1 / 2}\left(F=1 / 2, m_{F}=\right.$ $1 / 2)$ and $2 P_{1 / 2}\left(F=1 / 2, m_{F}=-1 / 2\right)$ as $|1\rangle,|2\rangle,|3\rangle$ and $|4\rangle$, respectively. The cold atoms are trapped in two state-selective optical potentials as shown in FIG 1 (c) and (d). We assume that the states $|1\rangle$ and $|2\rangle$ have the same the state-selective optical potential, say sublattice $A$, and $|4\rangle$ only perceives the other state-selective optical potential, say sublattice $B$. Here, for convenience, we assume that atoms in state $|3\rangle$ also perceive sublattice $A$. However, this is unnecessary in our scheme, for the population of the quantum state $|3\rangle$ is finally eliminated. The two sublattices have the lattice spacings $2 l_{x}$ and $l_{z}$ in the $x$ and $z$ directions, respectively. The two sublattices make up a $2 \mathrm{D}$ rectangular lattice with the lattice spacings $l_{x}$ and $l_{z}$, especially a $2 \mathrm{D}$ square lattice for $l_{x}=l_{z}$, when overlapping together as shown in FIG 1 (c) and (d). Without loss of generality, we suppose that atoms with internal states $|1\rangle$ and $|2\rangle$ are trapped in odd columns and ones with internal states $|4\rangle$ in even columns in the whole overlapped lattice. For convenience, we assume that the $2 \mathrm{D}$ square lattice considered here is in the $x-z$ plane as shown in FIG 1 (c). Two additional laser beams along the $y$ direction are added. When the potential barrier of the optical lattice along the $y$ direction is high enough, the tunneling along this direction between different planes is suppressed seriously, then every layer is an independent 2D lattice in $x-z$ plane.

Using $\{|1\rangle,|2\rangle,|3\rangle,|4\rangle\}$ as the basis, the Hamiltonian of free ultracold fermions in the optical lattice can be written in the second quantized form as follows,

$$
\hat{H}_{0}=\int d^{2} r \hat{\Psi}^{\dagger}\left(-\frac{\hbar^{2}}{2 m} \nabla^{2}+V(\mathbf{r})\right) \hat{\Psi}
$$

where $\hat{\Psi}^{\dagger}=\left(\hat{\Psi}_{1}^{\dagger}, \hat{\Psi}_{2}^{\dagger}, \hat{\Psi}_{3}^{\dagger}, \hat{\Psi}_{4}^{\dagger}\right)$ and $\hat{\Psi}=\left(\hat{\Psi}_{1}, \hat{\Psi}_{2}, \hat{\Psi}_{3}, \hat{\Psi}_{4}\right)^{T}$ ( $T$ denotes the matrix transposition) with $\hat{\Psi}_{i}(\mathbf{r})$ and $\hat{\Psi}_{i}^{\dagger}(\mathbf{r})$ being field operators corresponding to annihilating and creating an atom with the internal quantum state $|i\rangle(i=1,2,3,4)$ at coordinate position $\mathbf{r}$ respectively. Here, $V(\mathbf{r})$ is the trap potential matrix as

$$
V(\mathbf{r})=\left(\begin{array}{cccc}
V_{A}(\mathbf{r}) & 0 & 0 & 0 \\
0 & V_{A}(\mathbf{r}) & 0 & 0 \\
0 & 0 & V_{A}(\mathbf{r}) & 0 \\
0 & 0 & 0 & V_{B}(\mathbf{r})
\end{array}\right)
$$

where $V_{X}(\mathbf{r})(X=A, B)$ are the two state-selective periodic potentials. The ground state $|1\rangle$ is coupled to the excited state $|3\rangle$ via a laser field with the corresponding Rabi frequency $\Omega_{1} e^{-i q_{1} z}$ and the state $|2\rangle$ is coupled to the excited state $|3\rangle$ via a laser field with the corresponding Rabi frequency $\Omega_{2} e^{i q_{2} z}$ as shown in FIG.1(a) and (b).
The corresponding light-atom interaction Hamiltonian is,

$$
\hat{H}_{1}=\int d^{2} r \hat{\Psi}^{\dagger} M \hat{\Psi}
$$

with

$$
M=\hbar\left(\begin{array}{cccc}
0 & 0 & \Omega_{1} e^{i q_{1} z} & 0 \\
0 & 0 & \Omega_{2} e^{-i q_{2} z} & 0 \\
\Omega_{1} e^{-i q_{1} z} & \Omega_{2} e^{i q_{2} z} & 0 & 0 \\
0 & 0 & 0 & 0
\end{array}\right)
$$

where $\Omega_{j}(j=1,2)$ are the Rabi frequencies. Additionally, the quantum state $|1\rangle$ is coupled to the quantum state $|4\rangle$ via a laser field propagating in the $y$ direction with Rabi frequency $\Omega_{3} e^{i q_{3} y}$. Because $e^{i q_{3} y}$ is a constant in the $x-z$ plane, we can omit this phase factor by supposing the two-dimensional lattice on the $y=0$ plane. The corresponding interaction Hamiltonian is

$$
\hat{H}_{2}=\int d^{2} r \hat{\Psi}^{\dagger} N \hat{\Psi}
$$

with

$$
N=\hbar\left(\begin{array}{cccc}
0 & 0 & 0 & \Omega_{3} \\
0 & 0 & 0 & 0 \\
0 & 0 & 0 & 0 \\
\Omega_{3} & 0 & 0 & 0
\end{array}\right)
$$

The total Hamiltonian can be written as $\hat{H}=\hat{H}_{0}+\hat{H}_{1}+$ $\hat{H}_{2}$.

The Hamiltonian (3) can be diagonalized by the matrix,

$$
U=\left(\begin{array}{cccc}
\cos \theta & -\sin \theta e^{i q z} & 0 & 0 \\
\frac{\sqrt{2}}{2} \sin \theta e^{-i q z} & \frac{\sqrt{2}}{2} \cos \theta & -\frac{\sqrt{2}}{2} e^{-i q_{2} z} & 0 \\
\frac{\sqrt{2}}{2} \sin \theta e^{-i q z} & \frac{\sqrt{2}}{2} \cos \theta & \frac{\sqrt{2}}{2} e^{-i q_{2} z} & 0 \\
0 & 0 & 0 & 1
\end{array}\right)
$$

where $q=q_{1}+q_{2}$ and $\tan \theta=\left|\Omega_{1}\right| /\left|\Omega_{2}\right|$. Correspondingly, we obtain the dressed states as

$$
\begin{aligned}
& \left|\chi_{1}\right\rangle=\cos \theta|1\rangle-\sin \theta e^{i q z}|2\rangle \\
& \left|\chi_{2}\right\rangle=\frac{\sqrt{2}}{2} \sin \theta e^{-i q z}|1\rangle+\frac{\sqrt{2}}{2} \cos \theta|2\rangle-\frac{\sqrt{2}}{2} e^{-i q_{2} z} \mid 3\left(\chi_{9}\right) \\
& \left|\chi_{3}\right\rangle=\frac{\sqrt{2}}{2} \sin \theta e^{-i q z}|1\rangle+\frac{\sqrt{2}}{2} \cos \theta|2\rangle+\frac{\sqrt{2}}{2} e^{-i q_{2} z}|3\rangle(0) \\
& \left|\chi_{4}\right\rangle=|4\rangle,
\end{aligned}
$$

with the energy eigenvalues $E_{i}=(0,-\hbar \Omega, \hbar \Omega, 0)$ with $\Omega=\sqrt{\left|\Omega_{1}\right|^{2}+\left|\Omega_{2}\right|^{2}}$. Here, the state $\left|\chi_{1}\right\rangle$ is a so-called dark state, which does not contain the component of the excited atomic state $|3\rangle$, and $\left|\chi_{2}\right\rangle,\left|\chi_{3}\right\rangle$ are bright states. In the dressed state basis $\left\{\left|\chi_{1}\right\rangle,\left|\chi_{2}\right\rangle,\left|\chi_{3}\right\rangle,\left|\chi_{4}\right\rangle\right\}$, the vector field operator can be written as $\hat{\Phi}=$ $\left(\hat{\Phi}_{1}, \hat{\Phi}_{2}, \hat{\Phi}_{3}, \hat{\Phi}_{4}\right)^{T}=U\left(\hat{\Psi}_{1}, \hat{\Psi}_{2}, \hat{\Psi}_{3}, \hat{\Psi}_{4}\right)^{T}$, where $\hat{\Phi}_{j}(j=$ $1,2,3,4)$ represent destructing an atom in the dressed state $\left|\chi_{j}\right\rangle(j=1,2,3,4)$. Thus, the Hamiltonian can be rewritten as

$$
\hat{H}=\int d^{2} r \hat{\Phi}^{\dagger}\left[\frac{1}{2 m}(-i \hbar \nabla-\tilde{\mathbf{A}})^{2}+\tilde{V}(\mathbf{r})+\tilde{N}\right] \hat{\Phi}
$$


where $\tilde{\mathbf{A}}=i \hbar U \nabla U^{\dagger}, \tilde{V}(\mathbf{r})=U V(\mathbf{r}) U^{\dagger}+U M U^{\dagger}+$ $\frac{\hbar^{2}}{2 m}\left[\left(U \nabla U^{\dagger}\right)^{2}+\nabla U \cdot \nabla U^{\dagger}\right]$ and $\tilde{N}=U N U^{\dagger}$. We straight- forwardly calculate these matrices and obtain,

$$
\tilde{\mathbf{A}}=-\hbar \mathbf{e}_{z}\left(\begin{array}{cccc}
-q \sin ^{2} \theta & \frac{\sqrt{2}}{2} q \sin \theta \cos \theta e^{i q z} & \frac{\sqrt{2}}{2} q \sin \theta \cos \theta e^{i q z} & 0 \\
\frac{\sqrt{2}}{2} q \sin \theta \cos \theta e^{-i q z} & \frac{1}{2} q \sin ^{2} \theta+\frac{1}{2} q_{2} & \frac{1}{2} q \sin ^{2} \theta-\frac{1}{2} q_{2} & 0 \\
\frac{\sqrt{2}}{2} q \sin \theta \cos \theta e^{-i q z} & \frac{1}{2} q \sin ^{2} \theta-\frac{1}{2} q_{2} & \frac{1}{2} q \sin ^{2} \theta+\frac{1}{2} q_{2} & 0 \\
0 & 0 & 0 & 0
\end{array}\right)
$$

and

$$
\tilde{V}(\mathbf{r})=\left(\begin{array}{cccc}
V_{A}(\mathbf{r}) & 0 & 0 & 0 \\
0 & V_{A}(\mathbf{r})-\hbar \Omega & 0 & 0 \\
0 & 0 & V_{A}(\mathbf{r})+\hbar \Omega & 0 \\
0 & 0 & 0 & V_{B}(\mathbf{r})
\end{array}\right)
$$

and

$$
\tilde{N}=\hbar\left(\begin{array}{cccc}
0 & 0 & 0 & \Omega_{3} \cos \theta \\
0 & 0 & 0 & \frac{\sqrt{2}}{2} \Omega_{3} \sin \theta e^{-i q z} \\
0 & 0 & 0 & \frac{\sqrt{2}}{2} \Omega_{3} \sin \theta e^{-i q z} \\
\Omega_{3} \cos \theta & \frac{\sqrt{2}}{2} \Omega_{3} \sin \theta e^{i q z} & \frac{\sqrt{2}}{2} \Omega_{3} \sin \theta e^{i q z} & 0
\end{array}\right)
$$

In our scheme, we only consider the atoms in the dressed states $\left|\chi_{1}\right\rangle$ and $\left|\chi_{4}\right\rangle$. Thus, we have to adiabatically eliminate the populations of the dressed states $\left|\chi_{2}\right\rangle$ and $\left|\chi_{3}\right\rangle$ and to avoid the atoms decaying into these two dressed states. This can be realized in the steps. First, we start with the atoms in the atomic state $|1\rangle$ and $\Omega_{1}=0, \Omega_{3}=0$ with $\Omega_{2}$ finite, then slowly turn $\Omega_{1}$, we will end up with the atoms in the dressed state $\left|\chi_{1}\right\rangle$ [41]. During this process, the variation of $\Omega_{1}$ is slow enough to satisfy the adiabatic condition $\left|\left\langle\chi_{j}|\partial / \partial t| \chi_{1}\right\rangle\right| \ll\left|E_{j}-E_{1}\right| / \hbar=\Omega$ with $j=2,3$ [42, 43]]. In the second step, we adiabatically turn the Rabi frequency $\Omega_{3}$ on, we will end up with atoms in the dressed states $\left|\chi_{1}\right\rangle$ and $\left|\chi_{4}\right\rangle$. To avoid the atoms decaying into the dressed states $\left|\chi_{2}\right\rangle$ and $\left|\chi_{3}\right\rangle$, the adiabatic conditions $\frac{1}{2 m}\left|\tilde{\mathbf{A}}_{j 1}\right|^{2}=\frac{\hbar^{2}}{2 m} q^{2} \sin ^{2} \theta \cos ^{2} \theta \ll\left|E_{j}-E_{1}\right|=\hbar \Omega$ and $\left|\tilde{N}_{j 4}\right|=\frac{\sqrt{2}}{2} \hbar \Omega_{3} \sin \theta \ll\left|E_{j}-E_{4}\right|=\hbar \Omega$ for $j=2,3$ are satisfied. This is to say, the off-diagonal elements of the Hamiltonian are small enough to avoid the atoms decaying into the dressed states $\left|\chi_{2}\right\rangle$ and $\left|\chi_{3}\right\rangle$.

Since the atoms are only in the dressed states $\left|\chi_{1}\right\rangle$ and $\left|\chi_{4}\right\rangle$, we consider the reduced space with the dressed state basis $\left\{\left|\chi_{1}\right\rangle,\left|\chi_{4}\right\rangle\right\}$. Therefore, the total Hamiltonian can be reduced to

$$
\begin{aligned}
\hat{H} & =\int d^{2} r \hat{\Phi}_{1}^{\dagger}\left[\frac{1}{2 m}(-i \hbar \nabla-\mathbf{A})^{2}+V_{A}(\mathbf{r})\right] \hat{\Phi}_{1} \\
& +\int d^{2} r \hat{\Phi}_{4}^{\dagger}\left[-\frac{\hbar^{2}}{2 m} \nabla^{2}+V_{B}(\mathbf{r})\right] \hat{\Phi}_{4}
\end{aligned}
$$

$$
+\hbar \Omega_{e} \int d^{2} r\left(\hat{\Phi}_{4}^{\dagger} \hat{\Phi}_{1}+\hat{\Phi}_{1}^{\dagger} \hat{\Phi}_{4}\right)
$$

where $\Omega_{e}=\tilde{N}_{14} / \hbar=\Omega_{3} \cos \theta$ and the $U(1)$ adiabatic gauge potential $\mathbf{A}=\tilde{\mathbf{A}}_{11}=\hbar q \sin ^{2} \theta \mathbf{e}_{z}$.

\section{MASSLESS DIRAC FERMIONS}

Taking the tight-binding limit, we can superpose the Bloch states to get Wannier functions $w_{a}\left(\mathbf{r}-\mathbf{r}_{i}\right)$ and $w_{b}\left(\mathbf{r}-\mathbf{r}_{j}\right)$ for sublattice $A$ and $B$, respectively. In the present case, we can expand the field operator in the lowest band Wannier functions as, $\hat{\Phi}_{1}(\mathbf{r})=$ $\sum_{m(o d d), n} \hat{a}_{m, n} e^{\frac{i}{\hbar} \int_{0}^{\mathbf{r}_{m n}} \mathbf{A} \cdot d \mathbf{r}} w_{a}\left(\mathbf{r}-\mathbf{r}_{m n}\right)$ and $\hat{\Phi}_{4}(\mathbf{r})=$ $\sum_{m(\text { even }), n} \hat{b}_{m, n} w_{b}\left(\mathbf{r}-\mathbf{r}_{m n}\right)$. Substituting the above expression into Eq. (16), we can rewrite the Hamiltonian as follows,

$$
\begin{aligned}
\hat{H}= & -\sum_{(m(o d d), n)}\left[t_{b} \hat{b}_{m+1, n+1}^{\dagger} \hat{b}_{m+1}+t_{a} e^{i \gamma} \hat{a}_{m, n+1}^{\dagger} \hat{a}_{m, n}\right. \\
& \left.+2 t_{1} \hat{a}_{m, n}^{\dagger} \hat{b}_{m+1, n}+\text { H.c. }\right]+\hat{H}_{K}
\end{aligned}
$$

with $\quad H_{K}=\epsilon_{a} \sum_{(m(o d d), n)} \hat{a}_{m, n}^{\dagger} \hat{a}_{m, n} \quad+$ $\epsilon_{b} \sum_{(m(\text { even }), n)} \hat{b}_{m, n}^{\dagger} \hat{b}_{m, n}$. Here, the parameters have the following forms: $t_{a}=\int d^{2} r w_{a}^{*}\left(\mathbf{r}-\mathbf{r}_{m, n+1}\right)\left(-\hbar^{2} \nabla^{2} / 2 m+\right.$ $\left.V_{A}\right) w_{a}\left(\mathbf{r}-\mathbf{r}_{m n}\right), t_{b}=\int d^{2} r w_{b}^{*}\left(\mathbf{r}-\mathbf{r}_{m, n+1}\right)\left(-\hbar^{2} \nabla^{2} / 2 m+\right.$ $\left.V_{B}\right) w_{b}\left(\mathbf{r}-\mathbf{r}_{m n}\right), t_{1}=\Omega_{e} \int d^{2} r w_{b}^{*}\left(\mathbf{r}-\mathbf{r}_{m+1, n}\right) w_{a}\left(\mathbf{r}-\mathbf{r}_{m n}\right)$, $\epsilon_{a}=\int d^{2} r w_{a}^{*}\left(\mathbf{r}-\mathbf{r}_{m, n}\right)\left(-\hbar^{2} \nabla^{2} / 2 m+V_{A}\right) w_{a}\left(\mathbf{r}-\mathbf{r}_{m n}\right)$, 
$\epsilon_{b}=\int d^{2} r w_{b}^{*}\left(\mathbf{r}-\mathbf{r}_{m, n}\right)\left(-\hbar^{2} \nabla^{2} / 2 m+V_{B}\right) w_{b}\left(\mathbf{r}-\mathbf{r}_{m n}\right)$ and $\gamma=2 \pi \sin ^{2} \theta \hbar q l_{z}$ is the phase resulted from the adiabatic gauge potential. In our scheme, we consider $\epsilon_{a}=\epsilon_{b}$, so $\hat{H}_{K}$ in Eq. (17) can be dropped out as a constant term, which does not affect the physics considered here.

First, we consider that the ideal conditions $\gamma=\pi, t_{a}=$ $t_{b}=t_{1}=t$ and $l_{x}=l_{z}=l$ are satisfied. In experiments, these conditions can be achieved. Taking the Fourier transformation, $\hat{a}(\mathbf{k})=\sum_{(m(o d d), n)} \hat{a}_{m, n} \exp \left(-i \mathbf{k} \cdot \mathbf{r}_{m, n}\right)$ and $\hat{b}(\mathbf{k})=\sum_{(m(\text { even }), n)} \hat{b}_{m, n} \exp \left(-i \mathbf{k} \cdot \mathbf{r}_{m, n}\right)$, we obtain the total Hamiltonian as

$$
\begin{aligned}
\hat{H}= & -2 t \sum_{k}\left[\cos \left(k_{z} l\right) \hat{b}^{\dagger}(\mathbf{k}) \hat{b}(\mathbf{k})-\cos \left(k_{z} l\right) \hat{a}^{\dagger}(\mathbf{k}) \hat{a}(\mathbf{k})\right. \\
& \left.+\cos \left(k_{x} l\right) \hat{a}^{\dagger}(\mathbf{k}) \hat{b}(\mathbf{k})+\cos \left(k_{x} l\right) \hat{b}^{\dagger}(\mathbf{k}) \hat{a}(\mathbf{k})\right] .
\end{aligned}
$$

Diagonalizing the above Hamiltonian (18), we obtain the quasiparticle energy spectrum $E(\mathbf{k})=$ $2 s t \sqrt{\cos ^{2}\left(k_{x} l\right)+\cos ^{2}\left(k_{z} l\right)}$ with $s= \pm 1$ being the band index, which is similar to the spectrum of $\pi$ flux states in quantum spin liquids [44. This energy spectrum has two energy bands and contains four zero-energy Dirac points, where the conduction and valence bands intersect, in the first Brillouin zone at $\mathbf{K}_{1}=(\pi / 2 l, \pi / 2 l), \mathbf{K}_{2}=(-\pi / 2 l, \pi / 2 l), \mathbf{K}_{3}=$ $(-\pi / 2 l,-\pi 2 l), \mathbf{K}_{4}=(\pi / 2 l,-\pi / 2 l)$. Near the Dirac points, the energy dispersion has standard cone-like shape as shown in Fig 2 (a) and (b) and the spectrum is linear. The low-energy state dynamics are described by linearizing their spectrum about the degeneracy points and are modeled by massless relativistic fermions.

For simplicity, we only consider the part around the Dirac points $\mathbf{K}_{1}$, and the physics around the other Dirac points are similar. Setting $\mathbf{k}=\mathbf{K}_{1}+\mathbf{p}$, we linearize the Hamiltonian around the Dirac point $\mathbf{K}_{1}$ as, $\hat{H}=\hbar v_{0} \sum_{p}\left[p_{z} \hat{b}^{\dagger}(\mathbf{p}) \hat{b}(\mathbf{p})-p_{z} \hat{a}^{\dagger}(\mathbf{p}) \hat{a}(\mathbf{p})+p_{x} \hat{a}^{\dagger}(\mathbf{p}) \hat{b}(\mathbf{p})+\right.$ $\left.p_{x} \hat{b}^{\dagger}(\mathbf{p}) \hat{a}(\mathbf{p})\right]$ with $v_{0}=2 t l / \hbar$, which can be rewritten in coordinate space as, $\hat{H}=\int d^{2} r \hat{\eta}^{\dagger}(\mathbf{r}) \hat{\mathcal{H}} \hat{\eta}(\mathbf{r})$, where $\hat{\eta}=\left(\hat{\eta}_{b}, \hat{\eta}_{a}\right)^{T}$ with $\hat{\eta}_{b}(\mathbf{r})=\int d^{2} p e^{-i \mathbf{p} \cdot \mathbf{r}} \hat{b}(\mathbf{p})$ and $\hat{\eta}_{a}(\mathbf{r})=$ $\int d^{2} p e^{-i \mathbf{p} \cdot \mathbf{r}} \hat{a}(\mathbf{p})$. Here, $\hat{\mathcal{H}}$ is the single-particle Hamiltonian as $\hat{\mathcal{H}}=\hbar v_{0}\left(\hat{p}_{x} \sigma_{x}+\hat{p}_{z} \sigma_{z}\right)$, where $\sigma_{x}$ and $\sigma_{z}$ are Pauli matrixes. We obtain the eigenstates

$$
\phi_{\mathbf{p}}^{s}=\frac{1}{\sqrt{2}}\left(\begin{array}{l}
\cos \frac{\alpha}{2}+s \sin \frac{\alpha}{2} \\
s \cos \frac{\alpha}{2}-\sin \frac{\alpha}{2}
\end{array}\right) e^{i \mathbf{p} \cdot \mathbf{r}},
$$

where $s= \pm 1$ and $\tan \alpha=p_{z} / p_{x}$. The corresponding eigenenergies are $E^{s}(\mathbf{p})=s \hbar v_{0} p$ with $p=\sqrt{p_{x}^{2}+p_{z}^{2}}$. When the wave vector is $\mathbf{p}=p_{x} \hat{x}+p_{z} \hat{z}$, the corresponding group velocity and pseudospin vector are $\mathbf{v}_{g}=$ $s v_{0}\left(p_{x} \hat{x}+p_{z} \hat{z}\right) / p$ and $\mathbf{c}=\left(p_{x} \hat{x}+p_{z} \hat{z}\right) / p$, respectively. It is easy to find that the three vectors $\mathbf{v}_{g}$, $\mathbf{c}$ and $\mathbf{p}$ are collinear, i.e., they are parallel to each other. There is an intimate relation between the pseudospin and motion of the quasiparticle or quasihole: pseudospin can only be directed along the propagation direction (say, for quasiparticles) or only opposite to it (for quasiholes). As a result, quasiparticles or quasiholes exhibit a linear dispersion relation $E=\hbar v_{0} k$, as if they were massless relativistic particles but the role of the speed of light is played here by the Fermi velocity $v_{0}$.
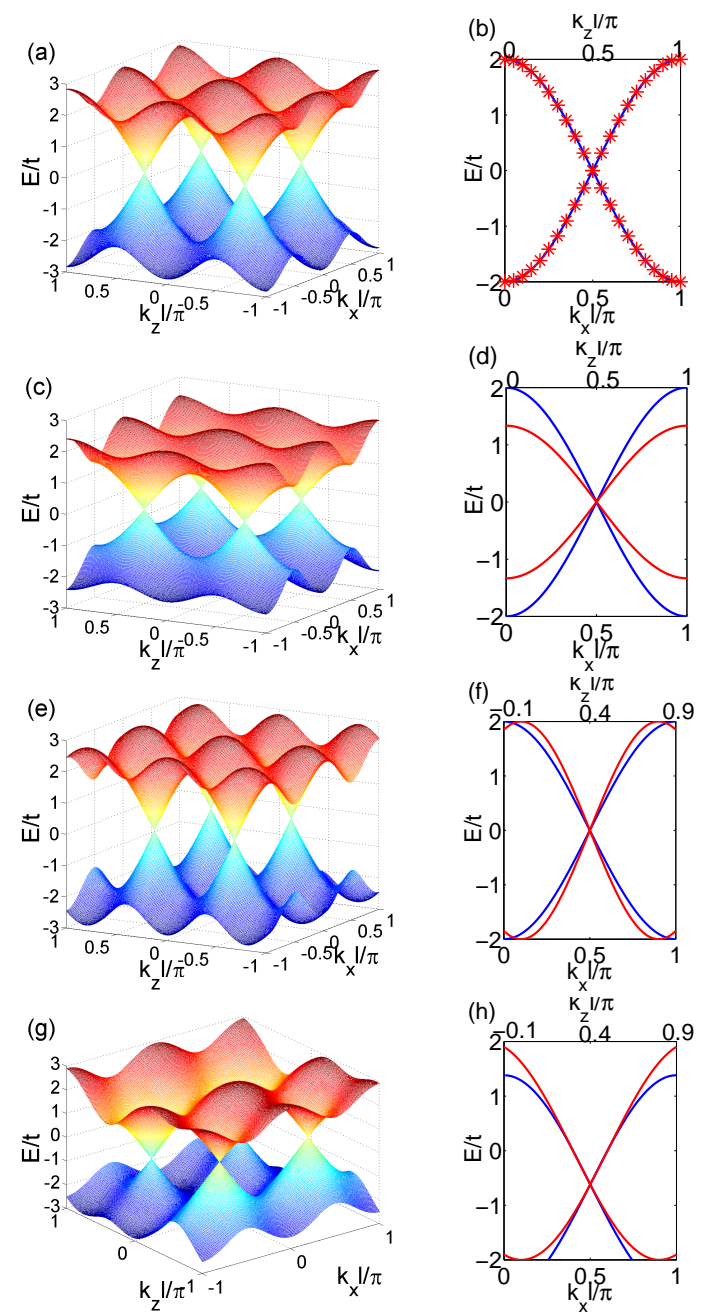

FIG. 2: Energy dispersion for cold fermionic atoms in a square optical lattice. (a) shows the energy dispersion and (b) represents the profiles of the energy dispersion with $k_{z}=\pi / 2 l$ (blue line) and $k_{x}=\pi / 2 l$ (red star), for the ideal case $t_{a}=$ $t_{b}=t_{1}=t, l_{x}=l_{z}=l, \gamma=\pi$. (c) shows the energy dispersion and (d) represents the profiles of the energy dispersion with $k_{z}=\pi / 2 l$ (blue line) and $k_{x}=\pi / 2 l$ (red line), for the anisotropic case $t_{a}=t_{b}=2 t_{1} / 3=2 t / 3, l_{x}=l_{z}=l, \gamma=\pi$. (e) shows the energy dispersion and (f) represents the profiles of the energy dispersion with $k_{z}=2 \pi / 5 l$ (blue line) and $k_{x}=\pi / 2 l$ (red line), for the anisotropic case $t_{a}=t_{b}=t_{1}=t$, $l_{z}=5 l_{x} / 4=5 l / 4, \gamma=\pi$. (g) shows the energy dispersion and (h) represents the profiles of the energy dispersion with $k_{z}=2 \pi / 5 l$ (blue line) and $k_{x}=\pi / 2 l$ (red line), for the anisotropic case with $t_{a}=t_{b}=t_{1}=t, l_{x}=l_{z}=l, \gamma=6 \pi / 5$.

In practice, the parameters may have fluctuations around the ideal conditions considered above. Fortunately, even the parameters deviate from the ideal ones, the massless Dirac fermion spectrum persists and remarkably exhibit anisotropic behaviors, which are just 
pursued in References [45] by adding external periodic potentials on graphene. Here, we provide alternative methods to exhibit anisotropic behaviors of massless Dirac fermions in a square optical lattice by setting the parameters deviated from the ideal situation.

For simplicity, we only consider three cases with the existence of parameter deviation from the ideal situation as follow: (i) $t_{a}=t_{b} \neq t_{1}=t, l_{z}=l_{x}=l$, $\gamma=\pi$; (ii) $t_{a}=t_{b}=t_{1}=t, l_{z} \neq l_{x}=l, \gamma=\pi$; (iii) $t_{a}=t_{b}=t_{1}=t, l_{x}=l_{z}=l, \gamma=\pi+\delta$ with $\delta \neq 0$. The corresponding dispersion relations are $E_{\mathrm{i}}(\mathbf{k})=2 s t \sqrt{\cos ^{2}\left(k_{x} l\right)+\left(t_{a} / t\right)^{2} \cos ^{2}\left(k_{z} l\right)}, E_{\mathrm{ii}}(\mathbf{k})=$ $2 s t \sqrt{\cos ^{2}\left(k_{x} l_{x}\right)+\cos ^{2}\left(k_{z} l_{z}\right)}$ and $E_{\mathrm{iii}}(\mathbf{k})=t\left[\cos \left(k_{z} l+\delta\right)-\right.$ $\left.\cos \left(k_{z} l\right)\right]+s t \sqrt{4 \cos ^{2}\left(k_{x} l\right)+\left[\cos \left(k_{z} l+\delta\right)+\cos \left(k_{z} l\right)\right]^{2}}$ for cases (i), (ii) and (iii), respectively, which are shown in FIG 2 (c)-(h). For case (ii), the four Dirac points are $\mathbf{K}_{1}=\left(\pi / 2 l_{x}, \pi / 2 l_{z}\right), \mathbf{K}_{2}=\left(-\pi / 2 l_{x}, \pi / 2 l_{z}\right), \mathbf{K}_{3}=$ $\left(-\pi / 2 l_{x},-\pi / 2 l_{z}\right), \mathbf{K}_{4}=\left(\pi / 2 l_{x},-\pi / 2 l_{z}\right)$, which are dependent on the lattice spacing in the $x$ and $z$ direction, while the Dirac points for cases (i) are the same as those of the ideal case. For case (iii), the four Dirac points are $\mathbf{K}_{1}=((\pi-\delta) / 2 l, \pi / 2 l), \mathbf{K}_{2}=$ $((-\pi-\delta) / 2 l, \pi / 2 l), \mathbf{K}_{3}=((-\pi-\delta) / 2 l,-\pi / 2 l), \mathbf{K}_{4}=$ $((\pi-\delta) / 2 l,-\pi / 2 l)$. Around the Dirac points, these spectra can be linearized as $E_{\mathrm{i}}^{s}(\mathbf{p})=s \hbar v_{0} p_{1}$ with $p_{1}=$ $\sqrt{p_{x}^{2}+f_{1}^{2} p_{z}^{2}}, E_{\mathrm{ii}}^{s}(\mathbf{p})=s \hbar v_{0} p_{1}$ with $p_{2}=\sqrt{p_{x}^{2}+f_{2}^{2} p_{z}^{2}}$ and $E_{\mathrm{iii}}^{s}(\mathbf{p})= \pm 2 t \sin (\delta / 2)+s \hbar v_{0} p_{3}$ with $p_{3}=\sqrt{p_{x}^{2}+f_{3}^{2} p_{z}^{2}}$, where $f_{1}=t_{a} / t=t_{b} / t, f_{2}=l_{z} / l$ and $f_{3}=\cos (\delta / 2)$. The corresponding single-particle Hamiltonian can be written as $\hat{\mathcal{H}}_{\mathrm{i}}=\hbar v_{0}\left(\hat{p}_{x} \sigma_{x}+f_{1} \hat{p}_{z} \sigma_{z}\right), \hat{\mathcal{H}}_{\mathrm{ii}}=\hbar v_{0}\left(\hat{p}_{x} \sigma_{x}+f_{2} \hat{p}_{z} \sigma_{z}\right)$ and $\hat{\mathcal{H}}_{\mathrm{iii}}= \pm 2 t \sin (\delta / 2)+\hbar v_{0}\left(\hat{p}_{x} \sigma_{x}+f_{3} \hat{p}_{z} \sigma_{z}\right)$ for cases (i),(ii),(iii), respectively. In all cases, the quasiparticles or quasiholes are still massless Dirac fermions and show chiral behavior. For the wave vector $\mathbf{p}=p_{x} \hat{x}+p_{z} \hat{z}$, the group velocity are pseudospin vector are $\mathbf{v}_{g}=s v_{t}\left(p_{x} \hat{x}+\right.$ $\left.f_{j}^{2} p_{z} \hat{z}\right) / p_{j}$ and $\mathbf{c}=\left(p_{x} \hat{x}+f_{j} p_{z} \hat{z}\right) / p_{j}$ for $j=1,2,3$. Here, the three vectors $\mathbf{v}_{g}, \mathbf{c}$ and $\mathbf{p}$ are not collinear and the dispersion relations near the Dirac points show anisotropic behaviors.

\section{BRAGG SPECTROSCOPY}

Here, we propose to identify massless Dirac fermionic quasiparticles with Bragg spectroscopy [46], which is extensively used to probe excitation spectra in condensed matter physics. In Bragg scattering, the atomic gas is exposed to two laser beams, with wavevectors $\mathbf{k}_{1}$ and $\mathbf{k}_{2}$ and a frequency difference $\omega$. The light-atom interaction Hamiltonian for Bragg scattering can be written as, $\hat{H}_{B}=\sum_{\mathbf{p}_{1}, \mathbf{p}_{2}} \hbar \Omega_{B} e^{-i \mathbf{q} \cdot \mathbf{r}}\left|\phi_{\mathbf{p}_{2}}^{f}\right\rangle\left\langle\phi_{\mathbf{p}_{1}}^{i}\right|+$ H.c. with $\mathbf{q}=\mathbf{p}_{2}-\mathbf{p}_{1}$, where the initial state $\left|\phi_{\mathbf{p}}^{i}\right\rangle$ is a filled state under Fermi surface and the final state $\left|\phi_{\mathbf{p}}^{f}\right\rangle$ is an empty state above Fermi surface. From the Fermi's golden rule,
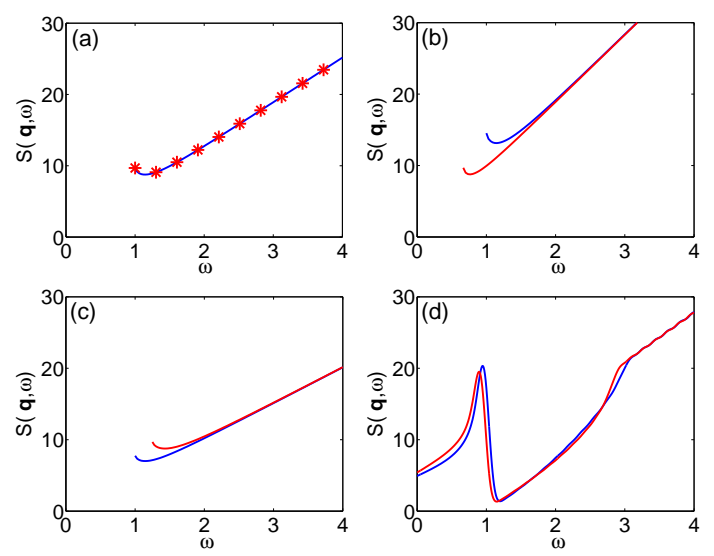

FIG. 3: The Bragg spectroscopies for the ideal case, (a) $t_{a}=$ $t_{b}=t_{1}=t, l_{x}=l_{z}=l, \gamma=\pi$, and the anisotropic cases, (b) $t_{a}=t_{b}=2 t_{1} / 3=2 t / 3, l_{x}=l_{z}=l, \gamma=\pi$, (c) $t_{a}=t_{b}=t_{1}=$ $t, l_{z}=5 l_{x} / 4=5 l / 4, \gamma=\pi$, (d) $t_{a}=t_{b}=t_{1}=t, l_{x}=l_{z}=l$, $\gamma=6 \pi / 5$ and $q=\pi / 10 l$. Here, we represent the Bragg spectroscopies with blue lines for the mentum difference $\mathbf{q}$ in the $x$ direction and with red stars or red lines for $\mathbf{q}$ in the $z$ direction. The frequency difference $\omega$ is expressed in units of $q v_{0}$ and the dynamic structure factor $S(\mathbf{q}, \omega)$ is expressed in units of $q / 8 \pi^{2} n v_{0}$ with $n$ being the number density of atoms in the system.

we obtain the dynamic structure factor as follows,

$$
\begin{aligned}
S(\mathbf{q}, \omega)= & \frac{1}{N \hbar^{2} \Omega_{B}^{2}} \sum_{\mathbf{p}}\left|\left\langle\phi_{\mathbf{p}+\mathbf{q}}^{f}\left|\hat{H}_{B}\right| \phi_{\mathbf{p}}^{i}\right\rangle\right|^{2} \\
& \times \delta\left(\hbar \omega-E_{\mathbf{p}+\mathbf{q}}^{f}+E_{\mathbf{p}}^{i}\right),
\end{aligned}
$$

where $N$ is the total number of atoms in the system.

Here, we consider the case of half filling of cold fermions in the optical lattice, i.e. the Fermi energy surface is at zero energy level, which is just at Dirac points for the cases except anisotropic case (iii). The numerical evaluation results of the dynamic structure factor is shown in FIG. 3. We note that there are lower cutoff frequencies $\omega_{r}$ for the fixed momentum difference $q$ and $S(\mathbf{q}, \omega)$ are approximately linear to the frequency difference $\omega$ for large frequency difference $\omega$ for FIG 3 (a),(b), (c). However, for FIG 3(d), the cutoff disappears for the Fermi surface is not at Dirac points for this case. FIG 3 (a) show that the bragg spectroscopy curves for the momentum difference $\mathbf{q}$ in the $x$ and $z$ directions are identical in the ideal case, which is just a consequence of the isotropy of the energy spectrum. From FIG 3 (b), (c) and (d), we clearly see that the Bragg spectroscopies are different for $\mathbf{q}$ in the $x$ and $z$ directions in the anisotropic cases, which features the anisotropic behaviors of those spectra.

\section{CONCLUSION}

In summary, we have proposed a novel scheme to realize massless Dirac fermions in a 2D square optical lat- 
tice with assistance of laser fields. For massless Dirac fermions, the gap is zero and the linear dispersion law holds. Our scheme is very robust against perturbations. Even the experimental situation deviates from the ideal conditions, massless Dirac fermions persist and, furthermore, exhibit novel features, i.e., anisotropic behaviors. Due to the absence of hexagonal symmetry, our scheme suggests a new direction to study Dirac fermions in the optical lattice.

\section{Acknowledgments}

This work was supported by the Teaching and Research Foundation for the Outstanding Young Faculty of Southeast University. X. J. Liu acknowledges support from US NSF Grant No. DMR-0547875 and ONR under Grant No. onr-n000140610122.
[1] G. W. Semenoff, Phys. Rev. Lett. 53, 2449 (1984).

[2] R. Jackiw, Phys. Rev. D 29, 2375 (1984).

[3] F. D. M. Haldane, Phys. Rev. Lett. 61, 2015 (1988).

[4] K. S. Novoselov, et al., Science 306, 666 (2004).

[5] K. S. Novoselov et al., Nature 438, 197 (2005).

[6] Y. Zhang, et al., Nature 438, 201 (2005).

[7] G. Li and E. Y. Andrei, Nature Phys. 3, 623 (2007).

[8] Y. Zheng and T. Ando, Phys. Rev. B 65, 245420 (2002).

[9] V. P. Gusynin et al., Phys. Rev. Lett. 95, 146801 (2005).

[10] C. Y. Hou et al., Phys. Rev. Lett. 98, 186809 (2007).

[11] R. Jackiw et al., Phys. Rev. Lett. 98, 266402 (2007).

[12] J. K. Pachos et al., Int. J. Mod. Phys. B, 21, 5113 (2007).

[13] P. H. Park and S. G. Louie, arXiv:0808.2127.

[14] S. L. Zhu et al., Phys. Rev. Lett. 98, 260402 (2007).

[15] E. Zhao et al., Phys. Rev. Lett. 97, 230404 (2006).

[16] L. B. Shao et al., arXiv:0804.1850.

[17] C. Wu et al., Phys. Rev. Lett. 99, 070401 (2007); C. Wu and S. Das Sarma, Phys. Rev. B 77, 235107 (2008).

[18] D. Jaksch et al., Phys. Rev. Lett. 81, 3108(1998).

[19] M. Greiner et al., Nature 415, 39, (2002).

[20] M. Lewenstein et al., Adv. Phys. 56, 243 (2007), and references therein.

[21] D. Jaksch and P. Zoller, New J. Phys. 5, 56 (2003).

[22] G. Juzeliūnas and P. Öhberg, Phys. Rev. Lett. 93, 033602 (2004).

[23] G. Juzeliūnas, P. Öhberg, J. Ruseckas, and A. Klein, Phys. Rev. A 71, 053614 (2005).

[24] G. Juzeliūnas, J. Ruseckas, P. Öhberg, and M. Fleischhauer, Phys. Rev. A 73, 025602 (2006).

[25] K. J. Günter, M. Cheneau, T. Yefsah, S. P. Rath, and J. Dalibard, Phys. Rev. A 79, 011604(R) (2009).

[26] R. Dum and M. Olshanii, Phys. Rev. Lett. 76, 1788 (1996).

[27] K. Osterloh, M. Baig, L. Santos, P. Zoller, and M. Lewenstein, Phys. Rev. Lett. 95, 010403 (2005).

[28] J. Ruseckas, G. Juzeliūnas, P. Öhberg, and M. Fleischhauer, Phys. Rev. Lett. 95, 010404 (2005).
[29] L. H. Lu and Y. Q. Li, Phys. Rev. A 76, 023410 (2007).

[30] Y. Li, C. Bruder, and C. P. Sun, Phys. Rev. Lett. 99, 130403 (2007).

[31] G. Juzeliūnas, J. Ruseckas, A. Jacob, L. Santos, and P. Öhberg, Phys. Rev. Lett. 100, 200405 (2008).

[32] A. Jacob, P. Öhberg, G. Juzeliūnas, and L. Santos, New J. Phys. 10, 045022(2008)

[33] X. J. Liu, X. Liu, L. C. Kwek, and C. H. Oh, Phys. Rev. Lett. 98, 026602 (2007).

[34] S. L. Zhu, H. Fu, C. J. Wu, S. C. Zhang, and L. M. Duan, Phys. Rev. Lett. 97, 240401 (2006).

[35] X. J. Liu, M. F. Borunda, X. Liu, and J. Sinova, Phys. Rev. Lett. 102, 046402 (2009).

[36] V. Pietilä and M. Möttönen, Phys. Rev. Lett. 102, 080403 (2009)

[37] J. Y. Vaishnav, J. Rusechas, C. W. Clark, and G. Juzeliūnas, Phys. Rev. Lett. 101, 265302 (2008).

[38] S. K. Dutta, B. K. Teo, and G. Raithel, Phys. Rev. Lett. 83. 1934 (1999).

[39] Y. J. Lin, R. L. Compton, A. R. Perry, W. D. Phillips, J. V. Porto, and I. B. Spielman, arXiv:0809.2976 (2008).

[40] J. Fuchs, G. J. Duffy, W. J. Rowlands, A. Lezama, P. Hannaford, and A. M. Akulshin, J. Phys. B: At. Mol. Opt. Phys. 40, 1117(2007).

[41] M. O. Scully and M. S. Zubairy, Quantum Optics, (Cambridge University Press, Cambridge, 1997).

[42] A. Messiah, Quantum Mechanics (North-Holland/ Elsevier Science, New York, 1962).

[43] J. M. Hou, L. J. Tian, and S. Jin, Phys. Rev. B 73, 134425 (2006).

[44] X. G. Wen, Quantum Field Theory of Many-Body Systems, (Oxford University Press, Oxford, 2004).

[45] C. H. Park et al., Nature Phys. 4, 213 (2008); C. H. Park et al., Phys. Rev. Lett. 101, 126804 (2008).

[46] D. M. Stamper-Kurn et al., Phys. Rev. Lett. 83, 2876 (1999). 